\title{
CINCO AÑOS DE EXPERIENCIA CON MISOPROSTOL INTRAVAGINAL PARA LA INDUCCION DE PARTO. USO DE UNA NUEVA PRESENTACION FARMACEUTICA EN SUPOSITORIOS*
}

\author{
Drs. Enrique Echeverría L. Edgardo Quiroz N., Juan Carlos Rozas A., Marcela Rocha O., \\ Carlos Hinrichs O., Rolando Jeréz M., Gloria Fernández C., ${ }^{* *}$ Lina Henríquez E.
}

Servicio de Obstetricia y Ginecología. Hospital Las Higueras de Talcahuano

${ }^{* *}$ Matronas

\section{RESUMEN}

Se presentan los resultados generales de 1534 casos con diversas patologías inducidos con misoprostol en tabletas, $50 \mu \mathrm{g}$ intravaginal cada 8 horas por dos veces al día, durante los años 1997 al 2001 en el Servicio de Obstetricia y Ginecología del Hospital Las Higueras de Talcahuano, destacando la baja incidencia de cesárea $(6,3 \%)$ y de Apgar bajo a los 5 minutos (0,5\%). En 47 casos se utilizó en forma prospectiva y randomizada para la inducción (enero a marzo de 2002), una nueva presentación farmacéutica (supositorios) aún no comercializada, la cual produjo un alto porcentaje de éxito a las dosis de $50 \mu \mathrm{g}$ cada 8 horas y $100 \mu \mathrm{g}$ cada 12 horas, hasta por un período de 48 horas: $96 \%$ y $81,8 \%$ respectivamente, con un bajo porcentaje de cesáreas $(7,1 \%)$ y de polisistolia $(6,4 \%)$ y ningún recién nacido deprimido.

\section{PALABRAS CLAVES: Inducción de parto con misoprostol}

\section{SUMMARY}

The results of labor induction in 1543 pathologic pregnancies from 1997 to 2001 with intravaginal misoprostol 50 micrograms $(\mu \mathrm{g})$, twice a day given every $8 \mathrm{hs}$ are analysed. A low section rate $(6.3 \%)$ and only $0.5 \%$ of Apgar score less than 7 at 5 min was obteined.

\section{KEY WORDS: Intravaginal misoprostol suppositories for induction of labor}

\section{INTRODUCCION}

El misoprostol, un análogo sintético de la prostaglandina $\mathrm{E} 1$, es sin lugar a dudas el medicamento más relevante aparecido en la farmacopea ginecoobstétrica de los últimos diez años. Inicialmente se utilizó a fines de los ochenta como agente gastroprotector para el tratamiento de la úlcera gastro- duodenal (1) por ser inhibidor de la secreción de HCL y estimulador de la secreción de moco y bicarbonato en la mucosa gástrica y duodenal, pero al poco andar se vio que era capaz de desencadenar contracciones uterinas por aumento de la entrada de calcio a la fibra muscular y favorecer la aparición de puentes intercelulares (2). Fue así como a principios de los noventa se usó como inductor de

*Trabajo recibido en mayo de 2002 y aceptado para publicación por el Comité Editor en junio de 2002. 
aborto con feto muerto primero y luego con feto vivo $(3,4)$. En la actualidad, desde la aparición del primer trabajo nacional en 1994 (5), se utiliza en nuestro país rutinariamente como inductor de parto en diversas patologías con feto de término, debido a la eficacia e inocuidad del medicamento (6, 7, 8, 9, 10, 11).

Nuestro Servicio empezó a usarlo el año 1993 en abortos inevitables y presentó su experiencia a la Sociedad de Obstetricia y Ginecología de la VIII Región. En 1995 inició su uso en embarazos en vías de prolongación y posteriormente en diversas patologías con fetos de término, buscando incluso la dosis óptima (12).

\section{MATERIAL Y METODO}

Con el objeto de mostrar el uso actual de misoprostol a la dosis de $50 \mu \mathrm{g}$ cada 8 horas intravaginal por dos veces (1 tableta $200 \mu \mathrm{g}$ ), para la inducción de parto en embarazos con diferentes patologías en la Maternidad del Hospital Las Higueras de Talcahuano, se revisan del Libro de Partos, los resultados de los partos producidos con esta técnica desde 1997 al 2001.

Además se efectúa un seguimiento prospectivo y randomizado por orden de ingreso al estudio entre los meses de enero y marzo de 2002, de la inducción de parto con dos diferentes dosis de misoprostol en forma de supositorios vaginales: 50 $\mu \mathrm{g}$ cada 8 horas y $100 \mu \mathrm{g}$ cada 12 horas, hasta por un período de 48 horas, (supositorios de 50 y $100 \mu \mathrm{g}$, una futura nueva forma comercial del medicamento: "Indupar", donados para este trabajo por un laboratorio privado*). Los supositorios fueron confeccionados con la dosis del medicamento ya indicada disueltos en una grasa saturada sintética inerte, llamada comercialmente withepfsol.

Todas las pacientes empezaron su inducción hospitalizadas en la sección de Alto Riesgo Obstétri$\mathrm{co}$, colocando mediante tacto vaginal, 50 microgramos de misoprostol ( $1 / 4$ de tabletas o 1 supositorio de 50 o de $100 \mu \mathrm{g}$ según el caso) en el fondo de saco vaginal posterior. Debían tener como requisito previo, una amnioscopia con líquido amniótico claro, un registro basal no estresante de los latidos cardiofetales reactivo, un útero sin cicatrices y una buena proporción pelvicofetal por estimación clínica y ecográfica. Luego permanecieron 2 horas en reposo en cama para permitir que el medicamento se disolviera y no se deslizara fuera de la vagina. Posteriormente se les permitió deambular libremente y fueron evaluadas por la matrona de la sección cada 60 minutos. Cuando estaban con 3 contrac- ciones en 10 minutos, se les envió a la sección de Preparto. Si a las 8 horas, no había aparecido dinámica de parto se colocaba una nueva dosis. En las inducciones con supositorio, usando el mismo criterio, se administraba otra dosis cada 8 ó 12 horas según el caso. $\mathrm{Si}$ en las primeras 24 horas no se lograba una dinámica efectiva, se repetía el mismo tratamiento al día siguiente, dándose por fracasada la inducción a las 48 horas.

Se consideró como tiempo de latencia, el transcurrido entre la última dosis y el inicio del trabajo de parto, desde ese momento hasta la dilatación completa, tiempo de dilatación y de ahí hasta la salida del feto, como tiempo de expulsivo.

Se estimó como éxito todos los casos en que el parto se produjo el primero o segundo día de inducción, independientemente si necesitó aceleración ocitócica posteriormente en el período de dilatación para mejorar la dinámica.

Todas las pacientes fueron informadas, de la utilización de una nueva forma farmacéutica de un medicamento ya probado para la inducción del parto.

Los resultados fueron procesados en Excel 3.0 en un PowerBook 145 Macintosh y redactados en Word 3.0.

\section{RESULTADOS}

En la Tabla I vemos la primera comunicación histórica que hizo nuestro servicio a la Sociedad de Obstetricia y Ginecología de la VIII Región en 1993, del uso de misoprostol vaginal en abortos inevitables, con $100 \%$ de éxito.

En la Tabla II están los resultados generales más relevantes obtenidos en 5 años de experiencia con el uso de misoprostol vaginal, con un porcentaje muy bajo de cesáreas $6,3 \%$ (incluso cuando se sospechó macrosomía: 10\%) y de RN con Apgar bajo a los 5 minutos $0,5 \%$.

Las patologías más frecuentes fueron: embarazo en vías de prolongación (EVP), síndrome hipertensivo del embarazo (SHE) y colestasis intrahepática del embarazo (CIE). El Pródromo de Parto ocupa el tercer lugar en frecuencia, ya que nuestro servicio tiene una conducta activa frente a él si no progresa espontáneamente en las primeras 24 horas de observación.

Las características generales de los 47 casos inducidos en forma randomizada con supositorios de misoprostol intravaginal desde enero a marzo de 2002 se observan en la Tabla III. Son todos embarazos de término, generalmente con un cuello no favorable (Bishop X: 5,3 ) y con diversas patologías (Tabla IV). 
Tabla I

INTERRUPCION DE EMBARAZOS DE II TRIMESTRE CON MISOPROSTOL*

\begin{tabular}{ll}
\hline & $(100 \mu$ g intravaginal)** \\
\hline Número de pacientes & 15 \\
Edad materna & $18-44$ años $(29,5)$ \\
Paridad & Primíparas $60 \%$ \\
Edad gestacional & $19-29$ semanas $(17)$ \\
Tiempo de inducción & $4-24$ horas $(10,12)$ \\
Exito & $100 \%$ \\
\hline
\end{tabular}

* 12 casos de huevo roto y 3 de aborto retenido.

${ }^{* *}$ La dosis se repite cada 10 horas.

En la Tabla V vemos las condiciones cervicales y los tiempos de latencia, dilatación y expulsivo muy parecidos para ambas dosis del medicamento ( $p$ NS). Hubo tres casos más con $50 \mu \mathrm{g}$, porque se nos acabaron los supositorios de $100 \mu \mathrm{g}$.

En la Tabla VI se observan los resultados más favorables con la dosis de $50 \mu \mathrm{g}$ tanto en las prime-

Tabla II

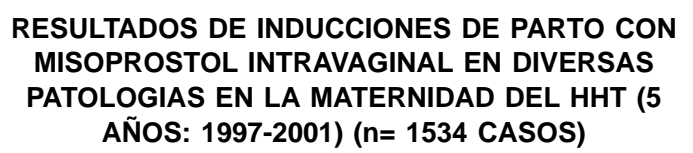

\begin{tabular}{|c|c|c|c|c|}
\hline $\begin{array}{c}\text { Patología } \\
n\end{array}$ & $\begin{array}{c}\text { Peso } \\
\bar{x}\end{array}$ & $\begin{array}{c}\text { Cesárea } \\
\%\end{array}$ & $\begin{array}{c}\text { Meconio } \\
\%\end{array}$ & Apgar $5^{\prime}<7$ \\
\hline $\begin{array}{l}\text { EVP (41s) } \\
\quad(562)\end{array}$ & 3587 & 6,2 & 18,7 & 0,2 \\
\hline $\begin{array}{l}\text { Pródromos } \\
\text { (344) }\end{array}$ & 3378 & 7 & 10,2 & 0,6 \\
\hline $\begin{array}{l}\text { SHE } \\
(198)\end{array}$ & 3328 & 6,6 & 8,1 & 0 \\
\hline $\begin{array}{c}\text { CIE } \\
(191)\end{array}$ & 3280 & 3,1 & 7,8 & 1 \\
\hline $\begin{array}{c}\text { DG } \\
(104)\end{array}$ & 3538 & 8,6 & 6,7 & 0 \\
\hline $\begin{array}{l}\text { Macrosomía } \\
\text { (40) }\end{array}$ & 3953 & 10 & 10 & 0 \\
\hline $\begin{array}{l}\mathrm{RCIU} \\
(32)\end{array}$ & 2607 & 3,1 & 6,3 & 0 \\
\hline $\begin{array}{l}\text { Obito } \\
(23)\end{array}$ & 2240 & 4,3 & 30,4 & 100 \\
\hline $\begin{array}{l}\text { Otro } \\
(36)\end{array}$ & 3099 & 11,1 & 11,8 & 2,8 \\
\hline $\begin{array}{l}\text { Total } \\
(1534)\end{array}$ & 4322 & 6,3 & 12,2 & 0,5 \\
\hline
\end{tabular}

Tabla III

\section{INDUCCION DE PARTO CON SUPOSITORIOS DE MISOPROSTOL INTRAVAGINAL. ENERO-MARZO $2002(n=47)$}

\begin{tabular}{ll}
\multicolumn{2}{c}{ Características generales de la población en estudio } \\
\hline Número de pacientes & (n) 47 \\
Edad & (x) 27 años; Rango 17 a 41 \\
Primigestas & $17(36,2 \%)$ \\
Edad gestacional & (x) 39 semanas; Rango 37 a 41 \\
Bishop & (x) 5,3 ; Rango 3 a 10
\end{tabular}

ras 24 horas (éxito de $88 \%$ versus $63,6 \%$ ), como a las 48 horas (éxito de $96 \%$ versus $81,8 \%$ ), a pesar de que con $50 \mu \mathrm{g}$ el porcentaje de cesáreas fue superior $12 \%$ versus $4,5 \%$ ( $p<0,05)$. Un bajo porcentaje necesitó aceleración ocitócica $26,2 \%$.

No se produjeron casos de Apgar bajo a los 5 minutos, ni se pesquisaron efectos colaterales indeseables en la madre y el recién nacido. Sólo hubo 3 casos de polisistolía (6,4\%), uno de ellos con síndrome de hiperestimulación $(2,1 \%)$, que terminó en cesárea.

\section{DISCUSION}

El alto porcentaje de éxitos ( $96 \%$ con $50 \mu \mathrm{g}$ cada 8 horas y $81,8 \%$ con $100 \mu$ g cada 12 horas) obtenidos a las 48 horas de inducción con esta nueva forma comercial de presentación, con una baja cantidad de cesáreas $(7,1 \%)$ y de polisistolías $(6,4 \%)$, sin recién nacidos deprimidos, produce una gran seguridad en su uso a esas dosis.

Si se hubiese utilizado una sola dosis de $50 \mu \mathrm{g}$ o de $100 \mu \mathrm{g}$ el éxito habría sido 44\% (11/25) y $36,4 \%(8 / 22)$, lo cual es significativamente más bajo que lo obtenido con la misma concentración de misoprostol vaginal en tabletas en dosis única, en otros trabajos del servicio: $77,3 \%(36 / 45)$ y $88 \%$

Tabla IV

\section{INDUCCION DE PARTO CON SUPOSITORIOS DE MISOPROSTOL INTRAVAGINAL. ENERO-MARZO $2002(n=47)$}

\begin{tabular}{lcc}
\hline Patología & $n$ & $\%$ \\
\hline EVP (41 semanas) & 17 & 36,2 \\
Pródromo & 14 & 29,8 \\
Patología ARO & 14 & 29,8 \\
Otras & 2 & 4,2 \\
\hline
\end{tabular}

EVP: (Embarazo en vía de prolongación). 
Tabla V

\section{INDUCCION DE PARTO CON SUPOSITORIOS DE MISOPROSTOL INTRAVAGINAL. ENERO-MARZO $2002(n=47)$}

\begin{tabular}{lcccc}
\hline \multicolumn{5}{c}{ Características de la inducción según dosis } \\
Dosis & $\begin{array}{c}\text { Bishop } \\
\bar{x}\end{array}$ & $\begin{array}{c}\text { T latencia } \\
\text { (minutos) }\end{array}$ & $\begin{array}{c}\text { T dilatación } \\
\text { (minutos) }\end{array}$ & $\begin{array}{c}T \text { expulsivo } \\
\text { (minutos) }\end{array}$ \\
\hline $\begin{array}{l}50 \mu \mathrm{g} \\
(25)\end{array}$ & 5,3 & 477 & 446 & 11 \\
$\begin{array}{l}100 \mu \mathrm{g} \\
(22)\end{array}$ & 5,3 & 562 & 461 & 8,7 \\
$\begin{array}{l}\text { Total } \\
(47)\end{array}$ & 5,3 & 514 & 453 & 9,8 \\
\hline
\end{tabular}

$(44 / 50)$ respectivamente $(p<0,05)$, con un tiempo total de inducción muy parecido, entre 10 y 14 horas. Esto hace suponer que la base de grasa saturada sintética inerte en que está disuelto el misoprostol en los supositorios, retarda la aparición de dinámica uterina efectiva.

AGRADECIMIENTOS: Los autores agradecen la colaboración de los médicos residentes y matronas de la sección de ARO y Preparto del Servicio, que controlaron y atendieron a las pacientes en estudio como al Laboratorio Pasteur por su donación.

\section{BIBLIOGRAFIA}

1. Willson D: Role of prostaglandins in gastroduodenal mucosal protection. J Clin Gastroenterol 1991; 13: 65-71.

2. Silverstein F, Saunders D: Misoprostol protecs the normal gastroduodenum against 7 days of aspirin. Gastroenterology 1988; 5: 428.

3. Misoprostol and legal medical abortion. Lancet 1991; 112: $1335-9$

4. Bugalho A, Bique C, Almeida L, Faúndez A: The effectiveness of intravaginal misoprostol (Cytotec) in inducing abortion after eleven weeks of pregnancy. Stud Fam Plann 1993; 24: 319-23.

5. Campos G, Guzmán S, Rodríguez J, Voto L, Margulles M: Misoprostol un análogo de la PG. El para la inducción de parto a término; estudio compa-
Tabla VI

\section{INDUCCION DE PARTO CON SUPOSITORIOS DE MISOPROSTOL INTRAVAGINAL. ENERO-MARZO $2002(n=47)$}

\begin{tabular}{|c|c|c|c|c|c|c|}
\hline \multicolumn{7}{|c|}{ Resultados inducción según dosis } \\
\hline Dosis & $\begin{array}{l}\text { Cesá- } \\
\text { rea (\%) }\end{array}$ & $\begin{array}{c}\text { Peso RN } \\
\text { (x) }\end{array}$ & $\begin{array}{c}\text { Meconio } \\
\text { (\%) }\end{array}$ & $\begin{array}{c}\text { \% éxito } \\
(24 \text { h) }\end{array}$ & $\begin{array}{c}\% \text { éxito } \\
(48 \text { h) }\end{array}$ & $\begin{array}{l}\% \text { uso } \\
\text { ocitoci. }\end{array}$ \\
\hline $\begin{array}{l}50 \mu \mathrm{g} \\
(25)\end{array}$ & 12 & 3477 & 8 & $\begin{array}{c}88 \\
(22 / 25)\end{array}$ & $\begin{array}{c}96 \\
(24 / 25)\end{array}$ & 28 \\
\hline $\begin{array}{l}100 \mu \mathrm{g} \\
(22)\end{array}$ & 4,5 & 3663 & 4,5 & $\begin{array}{c}63,6 \\
(14 / 22)\end{array}$ & $\begin{array}{c}81,8 \\
(18 / 22)\end{array}$ & 18,2 \\
\hline $\begin{array}{l}\text { Total } \\
(47)\end{array}$ & 7,1 & 3557 & 7,1 & $\begin{array}{c}76,6 \\
(36 / 47)\end{array}$ & $\begin{array}{c}89,4 \\
(42 / 47)\end{array}$ & 26,2 \\
\hline
\end{tabular}

rativo y randomizado con ocitocina. REV CHIL OBSTET GINECOL 1994; 59(3): 190-96.

6. Echeverría E, Rocha M: Estudio comparativo randomizado de inducción de parto con ocitocina y misoprostol en embarazos en vías de prolongación. Rev Chil Obstet Ginecol 1995; 60(2): 108-11

7. Ferrada $L$, Walton $R$, Ballesteros $S$, Ancapán $A$, Godoy G: Experiencia en un hospital de Chiloé, con el uso de prostaglandina sintética en la inducción del trabajo de parto en embarazos de término. REV CHIL OBStet GineCOl 1995; 60(6): 345-50.

8. Ferrada L, Walton R, Ancapán A y cols: Inducción del trabajo de parto con misoprostol intravaginal: experiencia de 5 años. Rev Chil Obstet Ginecol 1998; 63(1): 23-27.

9. Valderrama O, Ferrand P, Schwarze J y cols: Experiencia en el uso de misoprostol vaginal como inductor de trabajo de parto en pacientes en un servicio de alto riesgo obstétrico. REV CHIL OBSTET GINECOL 1998; 63(6): 431-36.

10. Echeverría E, Quiroz ME, Rozas JC y cols: Uso actual de misoprostol intravaginal para inducción de parto en diferentes patologías. REV CHIL OBSTET GINECOL 1999; 64(3): 217-20.

11. Domínguez C, Perucca E, Barrera S y cols: Inducción de parto en embarazos de alto riesgo obstétrico: misoprostol vaginal versus ocitocina. REV CHIL OBstet Ginecol 2000; 65(4): 262-68.

12. Echeverría E, Rocha M: Estudio comparativo de inducción de parto en embarazos en vías de prolongación con diferentes dosis de misoprostol. Rev Chil Obstet Ginecol 1996; 61(2): 101-04. 